\title{
Involvement of urinary bladder Connexin43 and the circadian clock in coordination of diurnal micturition rhythm
}

\author{
Hiromitsu Negoro1,2, Akihiro Kanematsu1,3, Masao Doi ${ }^{4}$, Sylvia O. Suadicani ${ }^{5,6}$, Masahiro Matsuo ${ }^{4}$, \\ Masaaki Imamura1, Takeshi Okinami ${ }^{1}$, Nobuyuki Nishikawa', Tomonori Oura7, Shigeyuki Matsui ${ }^{8}$, \\ Kazuyuki Seo ${ }^{4}$, Motomi Tainaka4 ${ }^{4}$ Shoichi Urabe ${ }^{4}$, Emi Kiyokage9 ${ }^{9}$ Takeshi Todo ${ }^{10}$, Hitoshi Okamura4, \\ Yasuhiko Tabata ${ }^{2} \&$ Osamu Ogawa ${ }^{1}$
}

Nocturnal enuresis in children and nocturia in the elderly are two highly prevalent clinical conditions characterized by a mismatch between urine production rate in the kidneys and storage in the urinary bladder during the sleep phase. Here we demonstrate, using a novel method for automated recording of mouse micturition, that connexin43, a bladder gap junction protein, is a negative regulator of functional bladder capacity. Bladder connexin43 levels and functional capacity show circadian oscillations in wild-type mice, but such rhythms are completely lost in Cry-null mice having a dysfunctional biological clock. Bladder muscle cells have an internal clock, and show oscillations of connexin43 and gap junction function. A clock regulator, Reverb $\alpha$, upregulates connexin43 transcription as a cofactor of Sp1, using Sp1 cis-elements of the promoter. Therefore, circadian oscillation of connexin43 is associated with the biological clock and contributes to diurnal changes in bladder capacity, which avoids disturbance of sleep by micturition.

\footnotetext{
${ }^{1}$ Department of Urology, Graduate School of Medicine, Kyoto University, Sakyo, Kyoto 606-8507, Japan. ${ }^{2}$ Department of Biomaterials, Institute for Frontier Medical Sciences, Kyoto University, Sakyo, Kyoto 606-8507, Japan. ${ }^{3}$ Department of Urology, Hyogo College of Medicine, Nishinomiya, Hyogo 663-8501, Japan. ${ }^{4}$ Department of Systems Biology, Graduate School of Pharmaceutical Sciences, Kyoto University, Sakyo, Kyoto 606-8501, Japan. 5 Department of Urology, Albert Einstein College of Medicine, Bronx, New York 10461, USA. 6 Dominick P. Purpura Department of Neuroscience, Albert Einstein College of Medicine, Bronx, New York 10461, USA. ${ }^{7}$ Department of Biostatistics, Kyoto University School of Public Health, Sakyo, Kyoto 606-8501, Japan. ${ }^{8}$ Department of Data Science, The Institute of Statistical Mathematics, Tachikawa, Tokyo 190-0014, Japan. ${ }^{9}$ Department of Anatomy, Kawasaki Medical School, Kurashiki, Okayama 701-0192, Japan. 10 Department of Radiation Biology and Medical Genetics, Graduate School of Medicine, Osaka University, Suita, Osaka 565-0871, Japan. Correspondence and requests for materials should be addressed to H.O. (e-mail: okamurah@pharm.kyoto-u.ac.jp) or to O.O. (email: ogawao@kuhp.kyoto-u.ac.jp).
} 
$\mathrm{N}$ octurnal enuresis is the involuntary loss of urine during sleep in childhood, and nocturia is the undesired waking at night for micturition later in life. Studies show that at least $10 \%$ of school children have nocturnal enuresis ${ }^{1,2}$, and $60-90 \%$ of the elderly, over 60 years, suffer from nocturia ${ }^{3,4}$. These conditions are detrimental to the quality of life by interfering with patients' selfesteem or sleeping habits, and they are the major diseases found in urology clinics. Nocturnal enuresis and nocturia are characterized by a mismatch between urine production rate in the kidneys, and storage in the urinary bladder ${ }^{5,6}$. During the sound sleep of a healthy person, a smaller volume of urine is produced than that during the daytime, and more urine is stored during the sleep phase than during the active phase ${ }^{7-9}$. Although it is unknown how such temporal variation is generated, these phenomena could be related to biological rhythms, because behaviour, physiology and metabolism in mammals are subject to a well-controlled daily rhythm, generated by an internal self-sustained molecular oscillator referred to as the circadian clock $^{10-13}$.

Circadian oscillations are driven by a transcription-translation feedback loop consisting of PER and CRY as negative components and CLOCK and BMAL1 as positive components. Rhythmic oscillations of this core loop are followed by the clock-associated oscillations of $D b p$ and Rev-erb $\alpha$, whose products regulate oscillations of a number of clock-controlled genes regulating cell- or organ-specific physiology through D-box and RORE sites, respectively ${ }^{14-16}$. These molecular oscillators exist in most body cells and organs ${ }^{13}$, but little is known about the role of the clock in urinary bladder function.

Micturition occurs by the contraction of smooth muscles of the urinary bladder on a sensation of fullness, which is precisely controlled by regulation of the central and peripheral nerves ${ }^{17,18}$. We and others have reported that an increase in connexin 43 ( $\mathrm{Cx} 43)$, a gap junction protein in the urinary bladder, enhances intercellular electrical and chemical transmission and sensitizes the response of bladder muscles to cholinergic neural stimuli. This results in a decrease in functional bladder capacity and an increase in micturition frequency in rats ${ }^{19-22}$. These phenomena mimic some aspects of an overactive bladder, a human pathological condition characterized by urinary urgency and increased micturition frequency ${ }^{23}$; however, the involvement of $\mathrm{Cx} 43$ in regulating normal bladder function remains unclear.

To further elucidate the role of $\mathrm{Cx} 43$ in bladder function, we investigated the effect of genetic ablation of $C x 43$ on micturition behaviour in mice and the implication of $C x 43$ for circadian micturition rhythm. The circadian micturition rhythm in free-moving mice still remains elusive, as the urine volume voided per micturition (UVVM) in mice is so minute (sometimes $<50 \mu \mathrm{l})^{24,25}$. To overcome this problem, we designed a novel system, called the automated voided stain on paper (aVSOP) method, which can accurately record micturition of mice for several days. Using this system, we demonstrated the role of $C \times 43$ and the circadian clock as regulators of functional bladder capacity in mice. We also showed that bladder muscle has internal rhythms of the clock and $\mathrm{Cx} 43$, which are correlated with oscillation in gap junction function. Further, we propose a novel paradigm that links the circadian clock with $\mathrm{Cx} 43$, in which Rev-erb $\alpha$ protein transactivates the $C \times 43$ promoter through interaction with Sp1.

\section{Results}

Cx43 is involved in control of functional bladder capacity. We began our study by developing an automated machine called aVSOP (Fig. 1a). The conversion of UVVM by mice from a drop area on filter paper has been reported to be an accurate method ${ }^{24,26}$, and this principle was applied to the automated system by using a laminated filter paper pre-treated to turn the edge of urine stains deep purple (Supplementary Fig. S1a). This modification enabled us to record the micturition of free-moving mice fed ad libitum for several successive days, for a UVVM as little as $10 \mu \mathrm{l}$ (Supplementary Fig. S1b).

To assess the effect of genetic Cx43 ablation on micturition, we compared heterozygous $C x 43^{+/-}$and wild-type (WT) $C \times 43^{+/+}$ littermate mice by the aVSOP method (Fig. 1b-d), because homozygous $C x 43^{-1-}$ mice die shortly after delivery ${ }^{27}$. Cx43 messenger RNA and protein levels in the urinary bladder of $C \times 43^{+/-}$ mice were decreased compared with those in $C \times 43^{+/+}$mice a

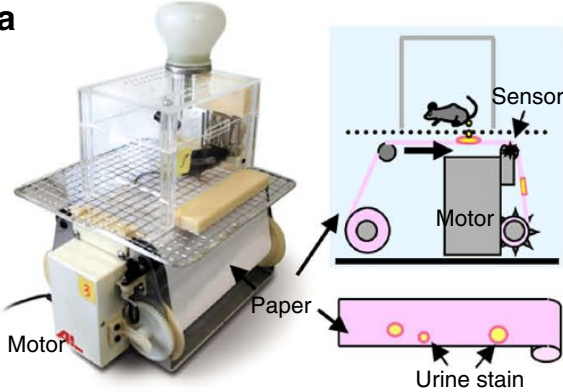

b

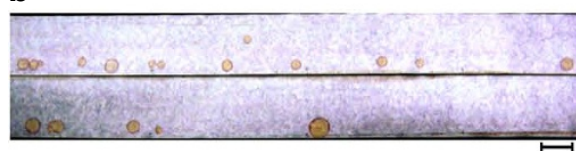

C
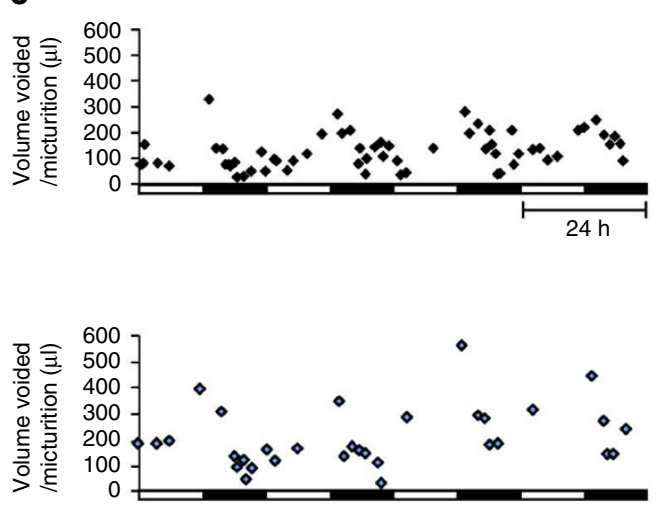

d

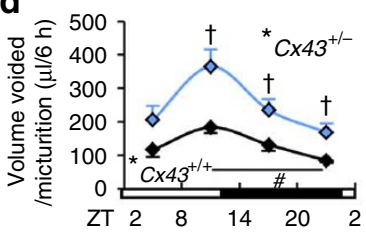

e

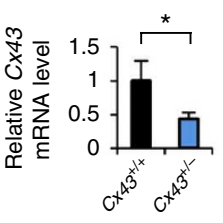

f

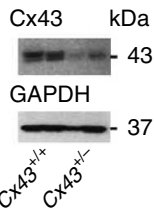

Figure 1 | aVSOP reveals an association between functional bladder capacity and $\mathbf{C x 4 3}$. (a) A photograph and diagram showing the aVSOP method. Each stain was traced, scanned and quantified by Image J 1.42 software. (b-d) Female $C \times 43^{+/-}$mice had larger functional bladder capacity than sex-matched $\mathrm{C} 43^{+/+}$littermates. (b) A photograph of urine spots on paper made by $\mathrm{C} \times 43^{+/+}$(top) and $\mathrm{C} \times 43^{+/-}$(bottom) mice. The scale bar indicates $10 \mathrm{~cm}$, corresponding to $1 \mathrm{~h}$. (c) Representative charts of UVVM of $\mathrm{C} \times 43^{+/+}$(top) and $C \times 43^{+/-}$(bottom) mice under LD conditions for 4 days. (d) UVVM per $6 \mathrm{~h}$ in $\mathrm{C}_{4} 43^{+/+}$and $\mathrm{C} \times 43^{+/-}$mice had diurnal variation (F(3[degrees of freedom (DF) for the time factor], $9\left[\right.$ error DF]) $=12.3$ and 10.9 , respectively; ${ }^{\star} P<0.005$ by one-way repeated measures ANOVA; $P<0.05$ in the late light (sleep) phase versus late dark (active) phase, followed by Bonferroni's post hoc test). Maximal correlations from a cosine curve (MaxCorr) of $\mathrm{C} \times 43^{+/+}$and $\mathrm{C} \times 43^{+/-}$mice were 0.949 and 0.989 , respectively: light-on at ZTO and off at ZT12. UVVM was significantly different between $C \times 43^{+/+}$and $C \times 43^{+/-}$mice $(F(1[D F$ for the strain factor],6[error DF]) $=11.2, P<0.05$ by two-way repeated measures ANOVA; $\uparrow P<0.05$ versus $C \times 43^{+/+}$by Bonferroni's post hoc test; $n=4$ for each group, with a total of 296 micturitions). Error bars represent s.e.m. (e) Relative $C \times 43$ mRNA levels of the urinary bladder in $\mathrm{C}_{4} 43^{+/-}$and $\mathrm{C} \times 43^{+/+}$mice used in the micturition analysis by real-time RT-PCR. Error bars represent s.d., $n=4$ for each mice. The value of $C \times 43^{+/+}$was set as $1 .{ }^{\star} P<0.05$ by Student's $t$-test. (f) $C \times 43$ protein expression of the urinary bladder in $C \times 43^{+/+}$and $C \times 43^{+/-}$mice. 
(Fig. 1e,f). In both genotypes, UVVM showed temporal variation under 12-hour light and 12-hour dark (LD) conditions (Fig. 1b-d), consistent with the nocturnal characteristics of the mice ${ }^{28}$. UVVM was higher in $C x 43^{+/-}$mice than that in $C x 43^{+/+}$mice (Fig. 1b-d), while total urine volume was not significantly different (Supplementary Fig. S1c).

These data demonstrate that the expression level of $\mathrm{Cx} 43$ is crucial for determining the functional capacity of the urinary bladder. This finding and the temporal variation in the micturition behaviour of the mice led us to focus on its association with $C \times 43$ expression in the bladder and the circadian clock.

Association of bladder clock with bladder capacity and Cx43. It has been reported that micturition shows a diurnal change in rodents as well as in humans $8,22,28,29$, although the day-night change is inverted because of the difference in diurnal versus nocturnal habits. However, it is unknown whether these changes are induced by an endogenous oscillator or only by a reflection of external LD changes. To address this in mice, we first examined micturition of WT mice under both LD and constant dark (DD) conditions. A distinct rhythm of UVVM was recorded, peaking at zeitgeber time (ZT) or circadian time (CT) 8-12 (Fig. 2a,b). $\chi^{2}$ analysis showed a circadian periodicity under both LD and DD conditions (Supplementary Fig. S2a). No significant difference between LD and
DD was found in the circadian amplitude ( 0.042 cycles per hour) quantified as relative power calculated by Fourier transform ${ }^{30}$ (Supplementary Fig. S2b). This demonstrates that the mouse could be a model animal for micturition rhythmicity, and that the rhythm could be related to an internal biological rhythm that is also operational in the absence of environmental change in LD cycles. The involvement of the circadian clock in micturition behaviour was tested in mice with a dysfunctional circadian clock by deletion of Cryptochrome-1 (Cry1) and Cryptochrome-2 (Cry2) (Cry-null mice); accordingly, these mice have completely arrhythmic behaviour and metabolism ${ }^{11,31}$. We placed our aVSOP system in a box with an infrared activity sensor and measured the micturition and locomotor activity, simultaneously. Similar to behavioural locomotor rhythms (Supplementary Fig. S3a), circadian characteristics of UVVM observed in WT mice were abolished in Cry-null mice (Fig. 2c,d) analysed by $\chi^{2}$ and Fourier transform (Supplementary Fig. S3b), as well as those of total urine volume and frequency (Supplementary Fig. S3c,d). These findings further support the notion that the circadian clock regulates UVVM.

We next examined whether the molecular machinery of the circadian clock is present in the urinary bladder. We found that the core oscillating machinery was present in the urinary bladder, as core clock genes including Per2 and Bmal1 (Fig. 2e), Per1, Cry1, Clock and Dbp (Supplementary Fig. S4) showed characteristic a

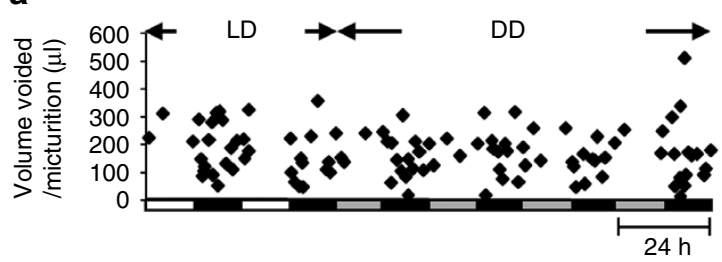

b

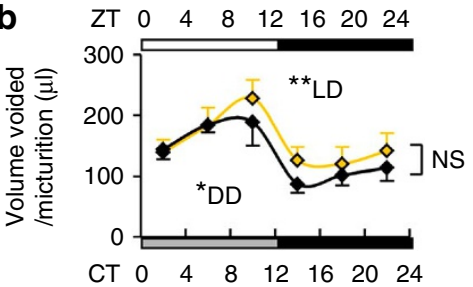

C

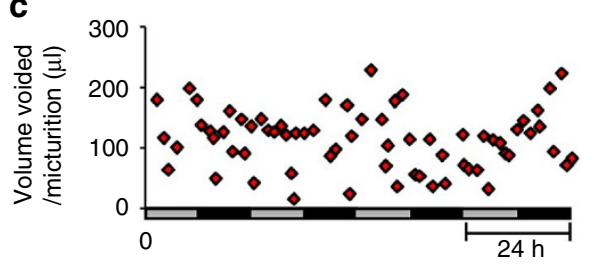

d

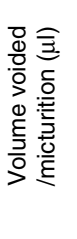

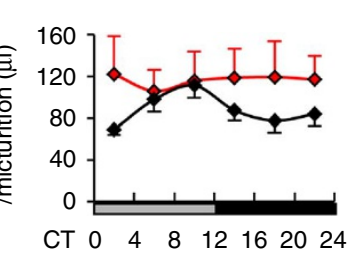

e
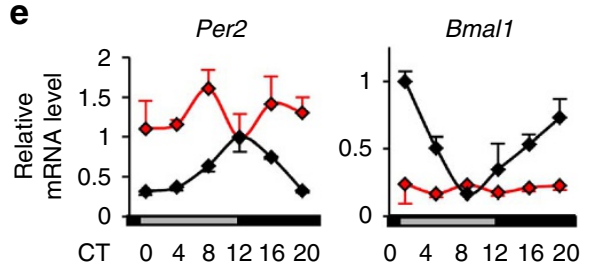

f

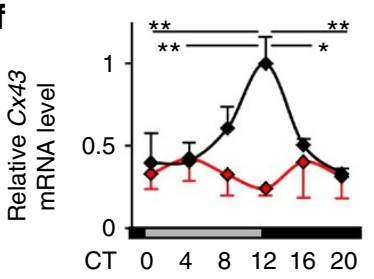

g

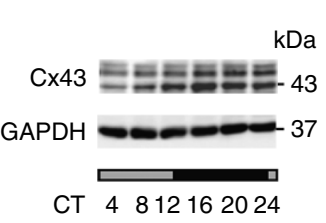

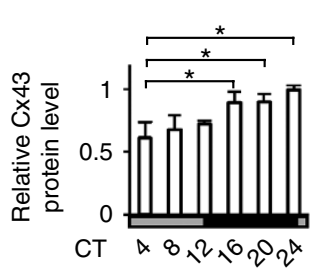

$\mathbf{h}$

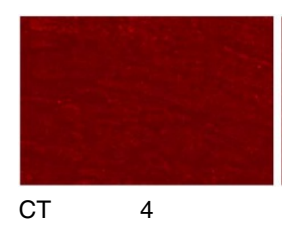

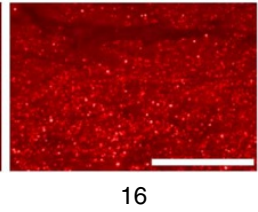

Figure 2 | Rhythmicity of micturition, clock genes and Cx43 expression in WT mice is disturbed in Cry-null mice. (a) A representative chart of UVVM of WT C57BL/6 mice under LD conditions followed by DD conditions. (b) Temporal UVVM every $4 \mathrm{~h}$ in WT mice $(n=5)$, for 8 days under LD conditions ( 940 micturitions) and 5 days under DD conditions (556 micturitions). Diurnal variation of UVVM in LD conditions $(F(5,20)=17.28$, ** $P<0.005$ by oneway repeated measures ANOVA) was also observed in DD conditions $\left(F(5,20)=8.23,{ }^{\star} P<0.05\right)$, with no significant difference among times in $L D$ versus DD by two-way repeated measures ANOVA. (c,d) Loss of circadian rhythm of UVVM in Cry-null mice under DD conditions. Age-matched female WT, 1,493 micturitions; Cry-null, 1,009 micturitions, $n=5$ each. (c) A representative chart of UVVM of Cry-null mice. (d) Temporal UVVM every $4 \mathrm{~h}$ in Cry-null (red-diamond) and WT (black-diamond) mice. Diurnal variation detected in WT mice $(F(5,20)=8.21, P<0.05$ by one-way repeated measures ANOVA) was not observed in Cry-null mice. (e) Temporal Per2, Bmal1 and (f) Cx43 mRNA accumulation in the bladder in WT and Cry-null mice $(n=3$ for each time point). MaxCorrs of Per2, Bmal1 and Cx43 were $(0.96,0.93$ and 0.85$)$ in WT and $(0.19,0.42$ and 0.38$)$ in Cry-null mice, respectively. There was no significant difference in temporal Cx43 mRNA levels in Cry-null mice by one-way ANOVA. (g) Immunoblots showing temporal changes in protein levels of Cx43 in WT-mouse bladder (three independent samples for each time point). (h) Immunostaining of the muscle layer in mouse urinary bladder showing a difference in immunoreactivity with a decrease in Cx43 at CT4 compared with CT16. Representative photographs of three replicated experiments with similar results are shown. Bar, $50 \mu \mathrm{m} .{ }^{\star} P<0.05$ and ${ }^{\star \star} P<0.01$ by one-way ANOVA with Tukey's post hoc test in $\mathbf{f}$ and $\mathbf{g}$. Error bars represent s.e.m. in $\mathbf{b}$ and $\mathbf{d}$, and s.d. in $\mathbf{e}-\mathbf{g}$. For the relative levels, the maximal values of WT were set as 1 in $\mathbf{e}$ and $\mathbf{f}$. F $(x, y), x=D F$ for the time factor; $y=$ error DF in $\mathbf{b}$ and $\mathbf{c}$. 
circadian expression profiles by real-time RT-PCR of circadian sampling of the urinary bladder every $4 \mathrm{~h}$ ( 6 time points of the day) in WT mice. Dysfunction of the bladder circadian clock in Cry-null mice was demonstrated by a disturbed rhythm of Per2 and Bmal1 (Fig. 2e). We performed DNA microarray analysis to investigate the genes showing circadian rhythm in the urinary bladder more extensively. Besides the clock genes, there are thousands of oscillating genes in the bladder, as in other organs ${ }^{32,33}$. Notably, our target gene, Cx43 (also known as Gja1), was among the 184 genes with clear circadian rhythmicity (defined as greater than the max correlation of 0.85 from the cosine curve with a 1.5 -fold amplitude of expression level ${ }^{34,35}$ ) (Supplementary Data 1).

Cx43 mRNA showed a clear circadian rhythm with a peak at CT12 and a trough at CT0 by real-time RT-PCR (Fig. 2f). Cx43 protein levels remained low during the sleep phase (CT4-12), but, began to elevate $4 \mathrm{~h}$ after the peak of mRNA expression, and formed a plateau during the active phase (CT16-24) (Fig. 2g). Immunostaining of $\mathrm{Cx} 43$ in the muscle layer of the urinary bladder at CT4 and at CT16 also showed a clear difference in immunoreactivity (Fig. 2h). In rats, in which day-night difference of micturition behaviour has been described $^{22,29}$, a similar correlation was observed between micturition rhythm (Fig. 3a), temporal variations of clock genes (Per2 and Bmal1) and Cx43 mRNA expressions in the urinary bladder (Fig. 3b), Cx43 protein levels (Fig. 3c) and Cx43 immunoreactivity (Fig. 3d).

The circadian change of mRNA was reflected in the protein level, and the increase/decrease in $\mathrm{Cx} 43$ protein expression correlated with the decrease/increase of UVVM, respectively, in WT mice and rats. Similarly, Cry-null mice tended to show a constantly low level of Cx43 mRNA (Fig. 2f) with a high level of UVVM (Fig. 2d). This inverse expression can be accounted for by altered bladder sensitivity caused by a differential level of gap junction formation by $\mathrm{Cx} 43$, because the half-life of connexin proteins is up to $5 \mathrm{~h}$ and expression largely follows transcript levels ${ }^{36,37}$. These findings suggest that the bladder circadian clock coordinates UVVM via circadian regulation of Cx43 gene expression.

Internal oscillations of bladder clock and $\mathrm{Cx} 43$ function. We investigated if the circadian clock oscillates in the bladder without systemic control. Therefore, we investigated its oscillation in ex vivo bladder in culture. We used mice carrying a PER2::LUC fusion protein, which has been engineered to produce bioluminescence, when the clock gene Per2 is activated in the presence of luciferin ${ }^{38}$. The ex vivo slice culture of the bladder from Per $2:: l u c$ mice demonstrated a robust oscillation of bioluminescence in the muscle layer of the bladder for at least four cycles (Fig. 4a; Supplementary Movie 1 ). The oscillation continued for $\sim 2$ months in the medium changed every 5 days. This oscillation was not observed in Per $2:: l u c$ mice with the Clock-mutation $\left(C l k^{\Delta 19} / C l k^{\Delta 19}\right)$ (Supplementary Fig. S5). These findings clearly demonstrate that a functional circadian clock exists in the smooth muscle of the urinary bladder.

We then examined cultured bladder smooth muscles cells (BSMC) under serum shock, an in vitro model of genetic oscillation ${ }^{39}$. After serum shock in BSMC, autonomous oscillation of clock genes (Per2 and Bmal1) was observed (Fig. 4b). Concurrently, Cx43 levels also showed autonomous rhythmicity (Fig. 4b), which was followed by a change in protein levels (Fig. 4c,d), as observed in the bladder in vivo (Figs $2 \mathrm{f}-\mathrm{h}$ and $3 \mathrm{~b}-\mathrm{d}$ ). This change occurred in parallel with a change in cell-cell communication rate as shown by a dyetransfer experiment with Lucifer yellow microinjected intracellularly (Fig. 4e). At $24 \mathrm{~h}$ after the initiation of rhythm when Cx43 protein levels were close to their nadir, the amount of dye-transferred cells was at its minimum. However, at $36 \mathrm{~h}$ when $\mathrm{Cx} 43$ protein levels were close to their peak, the amount of dye-transferred cells was considerably increased (more than twofold compared with the minimum level at $24 \mathrm{~h}$ ). These findings clearly demonstrate that bladder muscle cells have an internal rhythm generating system, which elicits oscillation in gap junction function.

Activation of $C x 43$ promoter by a clock component Rev-erb $\alpha$. We next investigated the molecular mechanism of $C x 43$ regulation by the clock. We searched for E-box, D-box and RORE sequences in the $5^{\prime}$ prime region of $C x 43$, because clock genes are known to regulate clock-controlled genes by binding to these sequences ${ }^{15,40,41}$. However, we identified no such canonical sequences in a speciesconserved manner within 10,000 bases from the transcription start site $^{\star}$ ( ${ }^{\star}$ for RORE, there are 3 atypical RORE sequences detected from -720 to -467 , but their transcriptional role for Cx43 was negated as shown in Supplementary Fig. S6 and Supplementary a

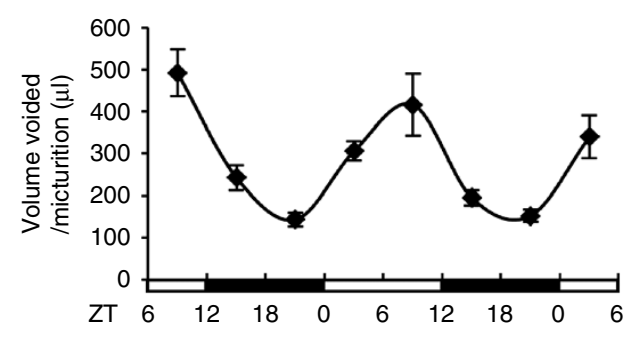

C

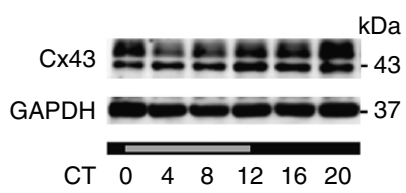

d

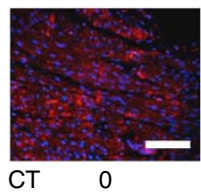

b

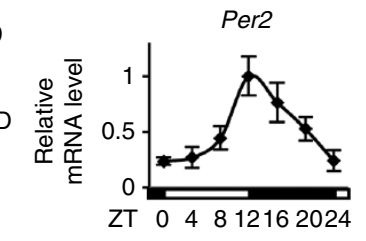

DD

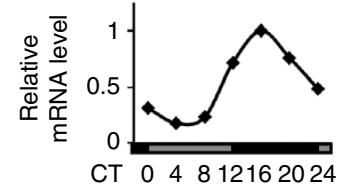

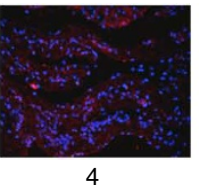
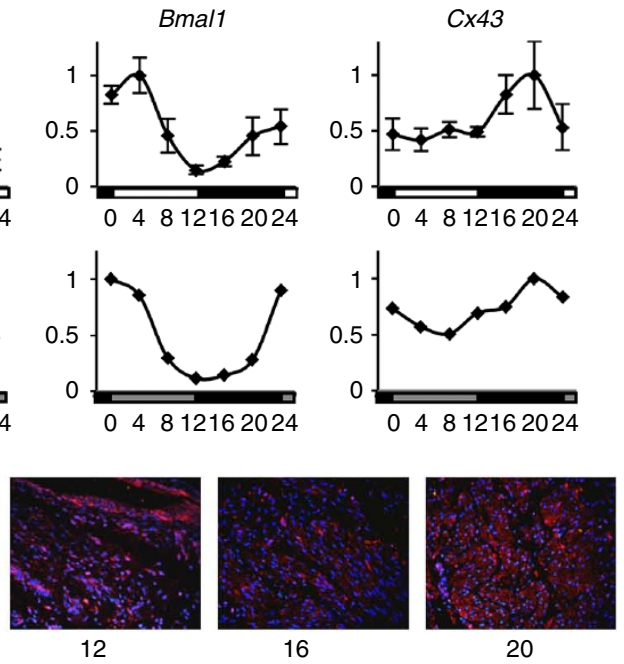

Figure 3 | Cx43 and clock-gene expression rhythms in rats and their correlation with micturition rhythm. (a) Patterns of UVVM in female SD rats under LD conditions for 2 days ( $n=15,1,001$ micturitions; $F\left(2.7\right.$ [degrees of freedom [DF] for the time factor],38.3[error DF]) $=11.9 ;{ }^{*} P<0.005$ by one-way repeated measures ANOVA with a Greenhouse-Geisser correction). (b) Temporal mRNA accumulation of Per2 Bmal1 and Cx43 in the rat bladder under LD and DD conditions ( $n=5$ and $n=2$ for each time point, respectively). MaxCorrs were $0.87,0.90$ and 0.84 in LD conditions, respectively, and $0.98,0.95$ and 0.93 in DD conditions, respectively. (c) Temporal Cx43 protein accumulation in the rat bladder under DD conditions as shown by immunoblotting. (d) Immunostaining of Cx43 in the rat bladder under DD conditions (red, Cx43; blue, DAPI). The scale bar indicates $100 \mu \mathrm{m}$. Error bars represent s.e.m. in $\mathbf{a}$, and s.d. in $\mathbf{b}$. For the relative expression, maximal values were set as 1 in $\mathbf{b}$. 
Discussion). Therefore, we suspected that the circadian clock might regulate the $C \times 43$ promoter by a novel mechanism.

We examined the effect of clock genes on the $C x 43$ promoter by promoter-reporter assays in HEK293T cells using a pGL-2-mouse Cx43-promoter-reporter construct containing - 1686/ + 165 (ref. 42). There was little effect of Clock/Bmall and Cry1 (positive and negative regulators of genes with E-box elements as shown for the Per1 promoter) or of Dbp and E4bp4 (regulators of genes with D-box
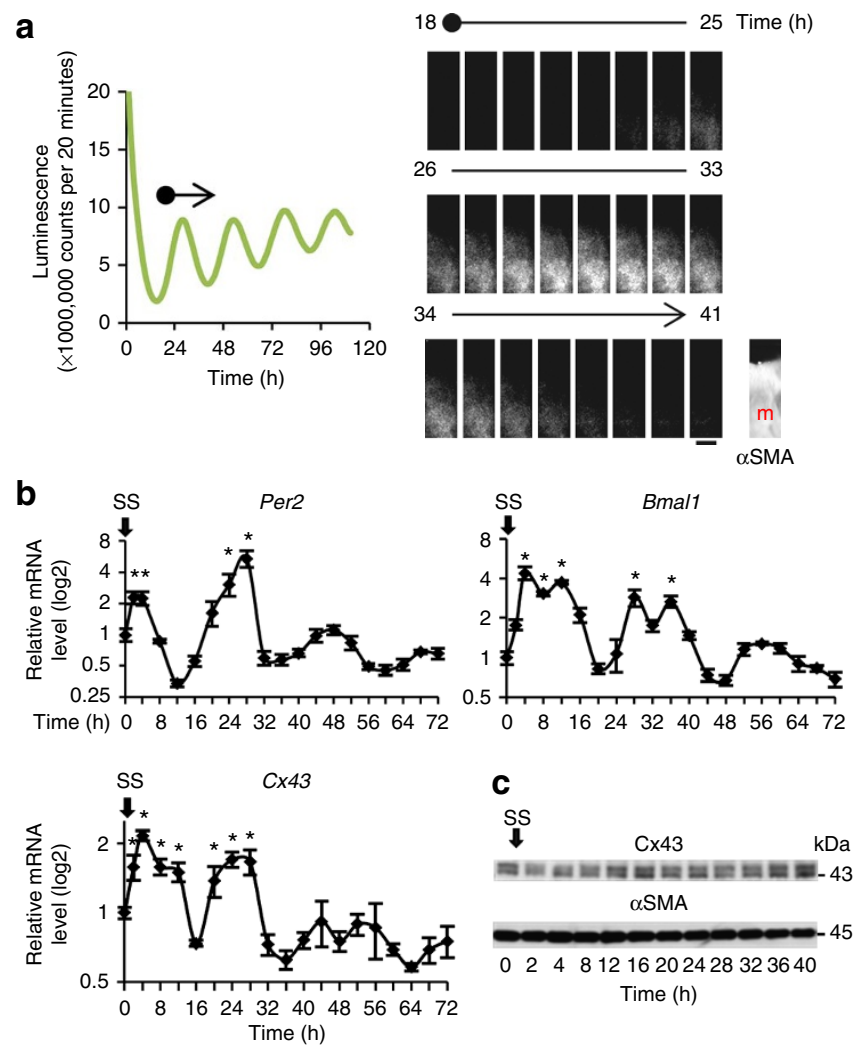

\section{d}

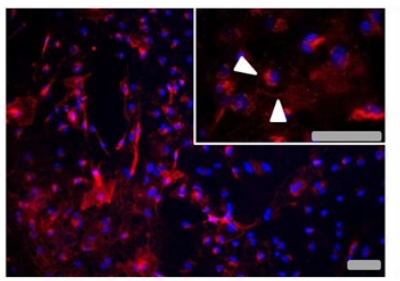

$12 \mathrm{~h}$

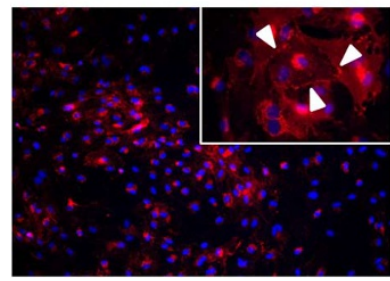

$36 \mathrm{~h}$

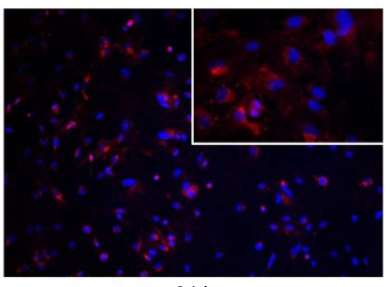

$24 \mathrm{~h}$

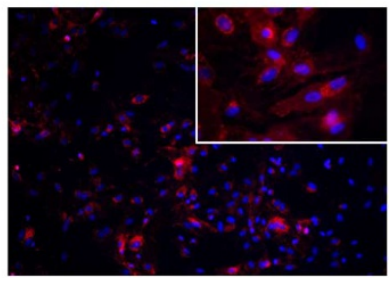

48
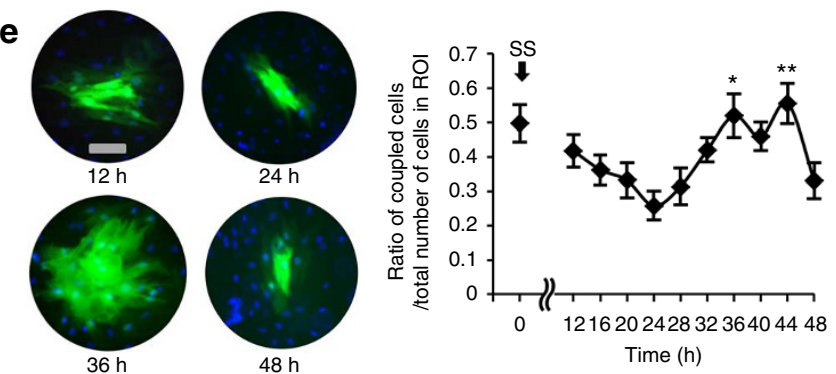

elements as shown for the Per1 promoter) on the Cx43 promoter (Supplementary Fig. S7a,b). A RORE-binding protein, Roro, also did not have any effect on Cx43 promoter activity. However, Reverb $\alpha$, which has been reported as a negative competitor of Ror $\alpha^{43}$ (as shown for the Bmal1 promoter, Supplementary Fig. S7c), markedly increased $C \times 43$ promoter activity (Supplementary Fig. S7a) in a dose-dependent manner (Fig. 5a), while the application of various concentrations of a mutant Rev-erb $\alpha$ (Rev-erb $\alpha$-truncated mutant deleted with 127-206 amino acids) failed to activate the Cx43 promoter (Fig. 5b).

Additionally, in BSMC, Rev-erbo dose-dependently upregulated Cx43 promoter activity (Fig. 5c). Conversely, inhibition of Rev-erb $\alpha$ by short interfering RNA (siRNA) decreased Cx43 mRNA and protein expression (Fig. 5d), while the same siRNA enhanced expression of Bmall. These results suggest that $C \times 43$ activation is elicited through an unreported positive transcriptional control by Rev-erb $\alpha$. Rev-erb $\alpha$ mRNA showed a clear circadian rhythm in mouse and rat urinary bladders with a peak time at CT/ZT 4-12 and a trough at CT/ZT16-24 (Fig. 5e,f). In Cry-null mice, Rev-erb $\alpha$ mRNA stayed arrhythmic at a lower level (Fig. 5e). These two patterns are consistent with the role of Rev-erb $\alpha$ as a positive regulator for $C x 43$, because the $C x 43$ expression profile showed a peak at CT/ZT12-20 and a trough at CT/ZT0-8 in WT mice and rats, but stayed arrhythmic at a lower level in Cry-null mice (Figs $2 \mathrm{f}$ and $3 \mathrm{~b}$ ).

Sp1-dependent activation of $C x 43$ transcription by Rev-erb $\alpha$. To clarify the novel molecular mechanism of Rev-erb $\alpha$ on promoter activity, we first attempted to identify the site with effects on the $C \times 43$ promoter by a deletion experiment because the $5^{\prime}$-region of $C \times 43$ contains several cis-elements including AP-1, AP-2, Sp1, half ERE, and cAMP-response element ${ }^{44}$. Truncated $-700 /+165,-300 /+165$ and $-147 /+165$ constructs did not affect the activation of the $C \times 43$ promoter by Rev-erb $\alpha$. In contrast, the transcription activation of Cx43 by Rev-erb $\alpha$ was markedly diminished in the $-54 /+165$ and $-44 /+165$ constructs (Fig. 6a).

Between -147 and -54 of the $C x 43$ promoter, there are ciselements evolutionally conserved among humans, rats and mice including three putative GC-rich Sp1-binding sites (Fig. 6b ${ }^{45,46}$.

Figure 4 | Oscillation of the circadian clock, Cx43 and gap junction function in bladder muscle cells without systemic control. (a) Oscillation of luminescence in bladder ex vivo slice culture obtained from $\mathrm{mPer} 2^{\text {Luciferase }}$ knock-in (Per2::/uc) mice. The period of oscillation was $24.92 \pm 0.56$ (mean \pm s.d.) $(n=10)$. The muscle layer of the bladder is shown by alpha smooth muscle actin ( $\alpha$ SMA) immunostaining. $m$, muscle. The scale bar indicates $100 \mu \mathrm{m}$. The oscillation of luminescence is also shown by a movie in Supplementary Movie 1. (b) Temporal variation of Per2, Bmal1 and Cx43 mRNA levels in serum-shocked rat BSMC. ${ }^{\star} P<0.01$ against the nadir of each genes' mRNA levels (time 12 for Per2, time 48 for Bmal1 and time 64 for $(x 43)$ by one-way ANOVA with Dunnett's post hoc test $(n=3-6)$. $\mathrm{SS}$, serum shock. For relative levels, the values before serum shock were set as 1. (c) Immunoblots showing temporal changes in $\mathrm{C} \times 43$ protein levels with $\alpha S M A$ as a loading control in serum-shocked rat BSMC. (d) Immunostaining of Cx43 at times 12, 24, 36 and $48 \mathrm{~h}$ in serum-shocked rat BSMC (red, Cx43; blue, DAPI). Arrow heads indicate typical plaques of gap junctions. Representative images of two replicate experiments with similar results are shown in $\mathbf{c}$ and $\mathbf{d}$. (e) Oscillation of gap junction function evaluated by Lucifer yellow microinjection in serum-shocked rat BSMC. One representative photograph of each time point (green, Lucifer yellow; blue, Hoechst 33342) and overall quantification of the degree of dye-coupling ( $n=6-9$, a total of 71 injections) is shown. ${ }^{\star} P<0.05$ and ${ }^{* \star} P<0.01$ versus time $24 \mathrm{~h}$ by one-way ANOVA with Tukey-Kramer's post hoc test. Similar significant differences were obtained in two independent experiments. Error bars represent s.e.m. in $\mathbf{b}$ and $\mathbf{e}$. Scale bars in $\mathbf{d}$ and $\mathbf{e}$ indicate $100 \mu \mathrm{m}$. 
a
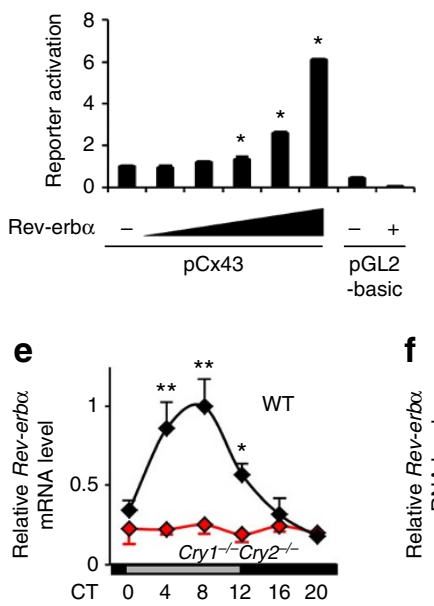

b

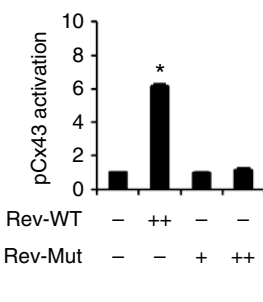

\section{f}

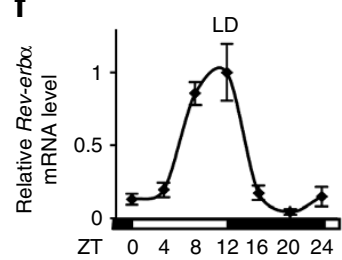

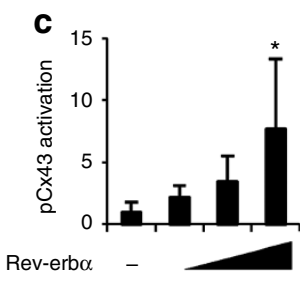

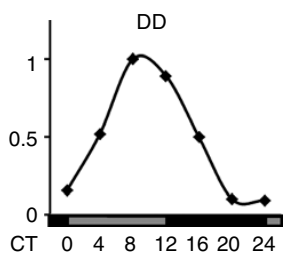

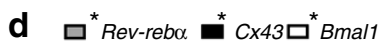
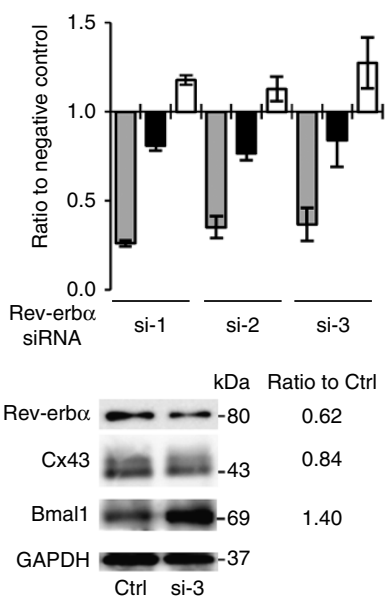

Figure 5 | Rev-erb $\alpha$ upregulates Cx43 expression. (a) Dose-dependent activation of $C x 43$ transcription by Rev-erb $\alpha$ in HEK293T cells ( $n=3$ for each dose). (b) Impaired activation of Cx43 transcription by a mutant of Rev-erb $\alpha$ without 127-206 amino acids from the N-terminal (Rev-Mut) in HEK293T cells ( $n=3$ for each dose). (c) Activation of $C \times 43$ transcription by Rev-erb $\alpha$ in rat BSMC ( $n=6$ for each dose). ${ }^{\star} P<0.01$ versus Rev-erb $\alpha(-)$ by one-way ANOVA with Dunnett's post hoc test in a-c. Similar data obtained in three independent experiments for $\mathbf{a}$ and $\mathbf{b}$, and in two independent experiments for c. (d) Suppression of Cx43 expression by knock-down of endogenous Rev-erb $\alpha$ in BSMC. Three types of Rev-erb $\alpha$ siRNAs, containing high (si-1), middle (si-2) and low (si-3) GC ratios or their controls containing corresponding GC ratios were transfected. $(n=4)$. mRNA and protein expression (data of si3) was normalized by $18 \mathrm{~s}$ ribosomal RNA and GAPDH, respectively. Interference of Rev-erb $\alpha$ mRNA significantly decreased mRNA expression of $C \times 43$ and increased Bmal1 compared with their corresponding controls (F(1[DF for the treatment factor],18[error DF]) $=324$ for Rev-erb $\alpha, 9.7$ for $C \times 43$ and 11.7 for Bmal1. ${ }^{\star} P<0.01$ by two-way ANOVA). Temporal bladder Rev-erb $\alpha$ mRNA accumulation in WT and Cry-null mice $(n=3)$. $(\mathbf{e})$ and in rats under LD $(n=5)$ and $\mathrm{DD}(n=2)$ conditions $(\mathbf{f}) .{ }^{\star} P<0.05$ versus $C T 8$ and ${ }^{\star *} P<0.01$ versus CTO, 16 and 20 in WT by one-way ANOVA with Tukey's post hoc test. No significant difference in Cry-null mice. MaxCorrs were WT, 0.98; Cry-null, 0.31; rats in LD, 0.84; in DD, 0.93. The maximal value of WT was set as 1. Error bars represent s.d. in a-f. For relative levels, Rev-erb $\alpha(-)$ was set as 1 in a-c.

Exogenous expression of a transcription factor $\mathrm{Sp} 1$ activated the Cx43 promoter in a dose-dependent manner, and its effect was dramatically increased by the addition of exogenous Rev-erb $\alpha$ (Fig. 6c). Protein expression of $\mathrm{Cx} 43$ was also upregulated by exogenous $\mathrm{Sp} 1$ and Rev-erb $\alpha$ (Fig. 6d). The promoter activation induced by $\mathrm{Sp} 1 / \mathrm{Rev}$-erbo was inhibited by mutations of the three Sp1 ciselements, in which the proximal was most crucial (Sp1C in Fig. 6e), and was completely abolished by deletion of all Sp1 sites (Fig. 6e). Sp3, another activator bound to Sp1 sites of Cx43 promoter ${ }^{45}$, also enhanced $C \times 43$ promoter activity in the presence of Rev-erbo (Supplementary Fig. S8), supporting the involvement of Sp1 sites for enhancement of $C \times 43$ transcription by Rev-erb $\alpha$.

In conclusion, unlike the negative transcriptional role of Reverb $\alpha$ using RORE sites, the novel positive transcriptional role of Rev-erb $\alpha$ requires $\mathrm{Sp} 1$ cis-elements on the Cx43 promoter.

Rhythmic assembly of Rev-erb $\alpha$ and Sp1 at $C x 43$ promoter. We hypothesized that Rev-erb $\alpha$ acts as a cofactor of Sp1, because Rev-erb $\alpha$ dose-dependently enhanced Cx43 activation by Sp1. To determine whether Rev-erbo interacts with Sp1 at Sp1 sites of the CX43 promoter, a co-immunoprecipitation assay and chromatin immunoprecipitation assay (ChIP) were performed in HEK293T cells transfected with HA-Rev-erb $\alpha$ and DDDDK-Sp1 (protein expression of these constructs are shown in Supplementary Fig. S9). We observed a DDDDK-Sp1 band in immunoprecipitates with an anti-HA antibody, and HA-Rev-erb $\alpha$ was detected in immunoprecipitates with an anti-DDDDK antibody (Fig. 7a), indicating that Rev-erb $\alpha$ can form a complex with Sp1. The ChIP assay revealed that, in immunoprecipitates of the chromatin fragments using antibodies for HA and DDDDK, specific enrichment was obtained by primers targeted on Sp1 cis-elements of the human Cx43 promoter (Fig. 7b). These findings demonstrate that Rev-erb $\alpha$ interacts with Sp1 at Sp1 sites of the CX43 promoter.
We next examined whether the endogenous Rev-erb $\alpha / S p 1$ complex is rhythmically formed on Sp1 sites of the Cx43 promoter. It is likely that Rev-erb $\alpha$, but not Sp1, could contribute to the rhythmic formation of the Rev-erb $\alpha / S p 1$ complex, because Sp1 mRNA showed no marked rhythm (Fig. 7c), in contrast with the strong rhythm of Rev-erb $\alpha$ mRNA in the bladder (Fig. 5e,f). To verify the rhythmic role of Rev-erb $\alpha$ estimated in vivo, we applied rat BSMC under serum shock in vitro for the ChIP assay on the Cx43 promoter using an anti-Rev-erb $\alpha$ antibody. After confirming the circadian expression of Rev-erb $\alpha$ mRNA and protein (Fig. 7d), we collected the cells before serum shock, and $14 \mathrm{~h}$ and $26 \mathrm{~h}$ after serum shock. At $26 \mathrm{~h}$ the level of Rev-erb $\alpha$ detected on Sp1 sites of the Cx43 promoter was higher than that at $14 \mathrm{~h}$ (Fig. $7 \mathrm{~d}$ ), when $C x 43$ mRNA expression showed a peak and nadir, respectively (Fig. 4b). Therefore, we conclude that this rhythmic formation of the Rev-erb $\alpha / \mathrm{Sp} 1$ complex on the Sp1 sites of Cx43 promoter is one mechanism that the clock induces for circadian oscillation of $C \times 43$ expression (Fig. 7e).

\section{Discussion}

Day-night micturition rhythm in humans enables a sound sleep during the sleep phase. This rhythm is not simply caused by a higher water intake during the day, because temporal variation in urine production is maintained in subjects under constant routine, when food and drink are taken equally during $24 \mathrm{~h}$ (ref. 47). In physiological conditions, nocturnal micturition is prevented not only by a decrease in urine production rate from the kidneys, but also by an increase in storage capacity of the urinary bladder ${ }^{7-9}$. Day-night differences in bladder capacity are experienced in the daily life of humans and their disturbance can be seen in disorders such as nocturnal enuresis and nocturia. The treatment of these patients in clinics is often limited to palliation because the precise mechanism underlying the micturition rhythm is unknown. 
a

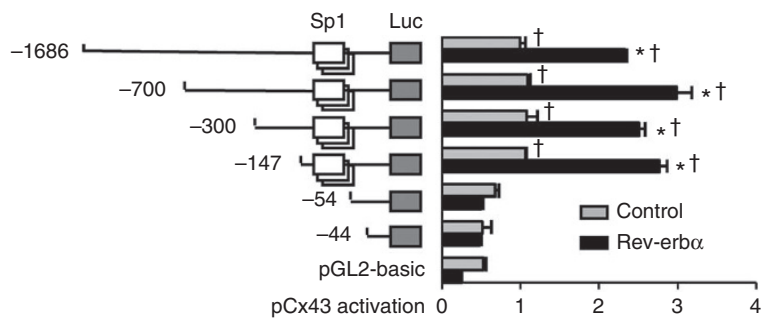

b
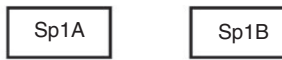

Sp1C

Human CCTCCTCCCAGCCIITCCCTITGCCCTCCCCTTTCTTCTAGCCCCTCCTCCCAGTTGAG

Rat CCTTCTCCCCGCCTITTCTTCCTCCCTCCCCTTTCTCCTGGCCCCTCCTTCCAGTTGAG

Mouse CCTCCTCCCCGCCIITTCTTCCTCCCTCCCCTTTCTCCTAGCCCCTCCTTCCAGTTGAG -40 *** ***** ****** $* * \overline{* * * * * * * * * * * * * * * * * \pi * * * * * *} * * * * * * * * *$
C

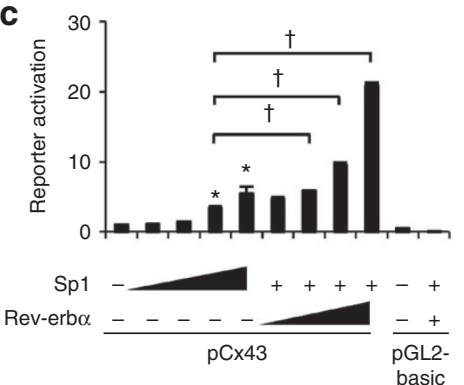

e

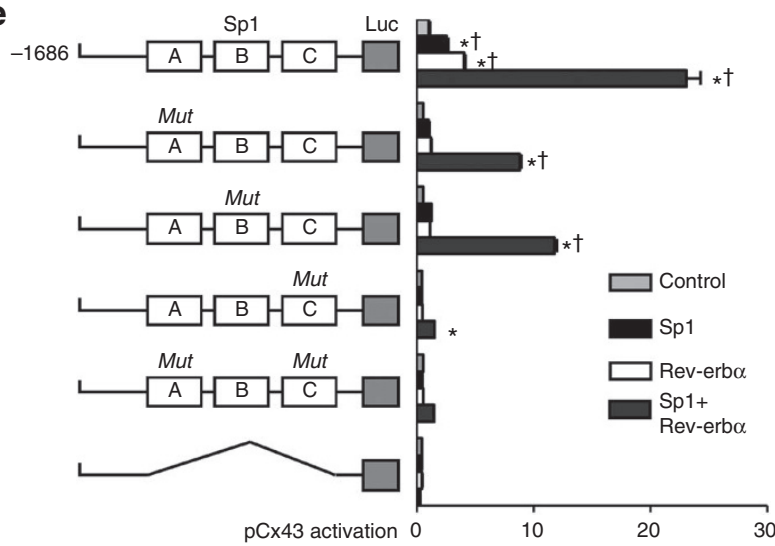

d

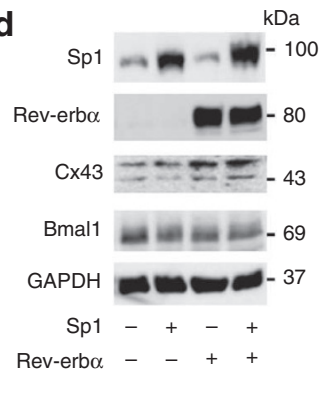

30
Figure 6 | Sp1-dependent activation of Cx43 expression by Rev-erb $\alpha$. (a) Sequences including Sp1 sites are indispensable for $\mathrm{Cx}_{x} 43$ promoter activation by Rev-erb $\alpha .{ }^{\star} P<0.001$ versus the control of each construct and $\uparrow P<0.001$ versus -54 (without Sp1 sequences) construct by two-way ANOVA with Bonferroni's post hoc test ( $n=3$ for each). (b-e) Rev-erb $\alpha$ and $\mathrm{Sp} 1$ activate $C_{x} 43$ expression using Sp1 sites. (b) Diagram of Cx43 promoter sequences including three Sp1 sites, labelled as Sp1A, $B$ and $C$. The asterisk indicates corresponding nucleotide sequences among humans, rats and mice. (c) Dose-dependent activation of $C_{x} 43$ transcription by Sp1 and Rev-erb $\alpha$ with Sp1. ${ }^{\star} P<0.001$ versus the value -without Sp1 and Rev-erb $\alpha$, and $\uparrow P<0.001$ by one-way ANOVA with Tukey's post hoc test ( $n=3$ for each). (d) Immunoblot analysis of the effect of Sp1 and Rev-erb $\alpha$ on expression of Cx43 and Bmal1 (control of negative regulatory effect by Rev-erb $\alpha$ ). (e) Impaired activation of $\mathrm{pC} \times 43$ with the Sp1 sites mutation by Sp1 and Rev-erb $\alpha .{ }^{\star} P<0.001$ versus the controls of each construct, and $\uparrow P<0.001$ versus the MutC construct by two-way ANOVA with Bonferroni's post hoc test ( $n=3$ for each group). Error bars represent s.d. in a, c and e. Cells used were HEK293T in all transfection experiments. The control without Rev-erbo and Sp1 was set as 1 in a, c and $\mathbf{e}$. One representative of two experiments with similar results is shown in $\mathbf{a}, \mathbf{c}, \mathbf{d}$ and $\mathbf{e}$.
In the beginning of the current study, we aimed to use a mouse model with/without genetic modification for investigating this mechanism. A day-night difference in micturition is known to exist in humans and rodents $8,22,28,29$, but circadian analysis of micturition in mice has been precluded by the difficulty in diachronic measuring of urine volume with high accuracy. To overcome these problems, we designed the aVSOP system, which enabled an accurate record of minute micturition volume as little as $10 \mu \mathrm{l}$ for several days. This method clearly recorded circadian rhythm of micturition in mice (also avoiding micturition during the sleep phase), suggesting that this rhythm should have a mechanism conserved among species. The aVSOP method also enabled us to use genetically engineered mice for unravelling the molecular events responsible for controlling micturition. In $C x 43^{+/-}$mice, this method showed the importance of $\mathrm{Cx} 43$ for determining functional bladder capacity, and in Cry-null mice, it showed a novel function of the circadian clock: circadian regulation of functional bladder capacity. Oscillation of the internal clock was demonstrated in the urinary bladder muscle layer ex vivo and in cultured BSMC, in parallel with the functional change in gap junctions, compatible with the rhythm of $\mathrm{Cx} 43$ levels. Collectively, these findings constitute the key concept of this study: the clock elicits oscillation in Cx43 levels and sensitivity of BSMC, which contribute to altered functional bladder capacity and micturition frequency during the 24-h cycle.

Micturition during the sleep phase is undesirable for humans, in terms of arousal from sleep, hygiene or maintenance of body temperature, which could also be applicable to rodents. By focussing on $\mathrm{Cx} 43$ in the urinary bladder and the circadian clock in the present study, we showed a novel aspect in normal and pathological physiology of the diurnal micturition rhythm. However, we should also note that $\mathrm{Cx} 43$ in the bladder is not the only determinant of this rhythm. Enuresis and nocturia are caused not only by decreased functional bladder capacity, but also by impairment of cortical arousal level in the brain, urine production rhythm in the kidneys ${ }^{4}$, and the interaction between each other ${ }^{48}$. In the bladder, there are also many other candidate molecules with diurnal oscillation, as listed in our microarray data (Supplementary Data 1), such as genes associated with smooth muscle contraction (Cacna1g, Ednrb and Gucyla3) or response to pain (Slc6a2, Ednrb and Grik1). These genes could also be contributing to micturition rhythm. Future studies may elucidate the association of these factors with the circadian clock, and the aVSOP method could serve as an important tool for that purpose.

The molecular mechanism of Cx43 oscillation that may underlie these phenomena is also noteworthy. Rev-erbo protein, known as a constitutive repressor ${ }^{43,49}$, acts as a transcriptional activator for $C \times 43$ by luciferase-reporter assay. Intriguingly, for $C \times 43$ activation, Rev-erb $\alpha$ does not require a canonical RORE site, but acts indirectly on Sp1 sites by interacting with Sp1 protein. This is similar to previous reports showing the physical interaction of $\mathrm{Sp} 1$ and nuclear receptors, such as RAR, RXR, ERs and PPAR $\gamma^{50-52}$. The identification of a co-activator-like function of Rev-erb $\alpha$ advocates a novel paradigm for controlling circadian gene expression: circadian clock components can modulate the activity of transcription factors coded by non-clock genes by functioning as transcriptional cofactors. Genome-wide screening of putative clock-associated sequences has revealed that many genes showing circadian oscillation do not have binding sequences for clock proteins ${ }^{14,15}$, and our finding suggests that these genes could possibly be regulated by clock proteins acting as cofactors.

In summary, the circadian clock is associated with the oscillating expression of $\mathrm{Cx} 43$ in BSMC via a previously unknown regulatory mechanism, and it contributes to changes in bladder capacity with an increase during the sleep phase and a decrease during the active phase. This study warrants chronobiological approaches for the investigation and treatment of nocturnal enuresis and nocturia. 
a

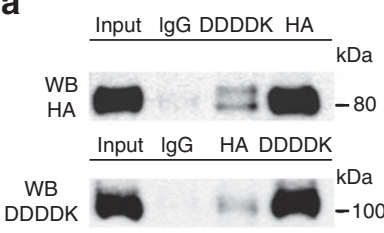

b

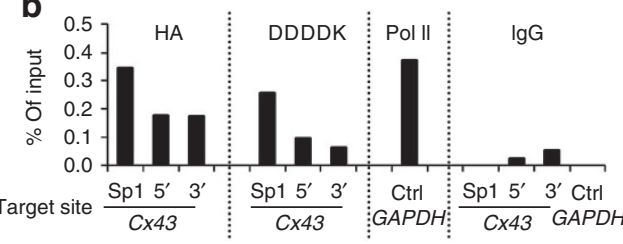

C

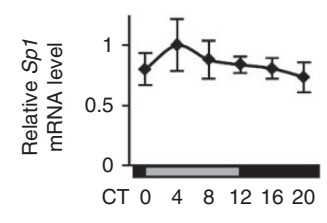

d
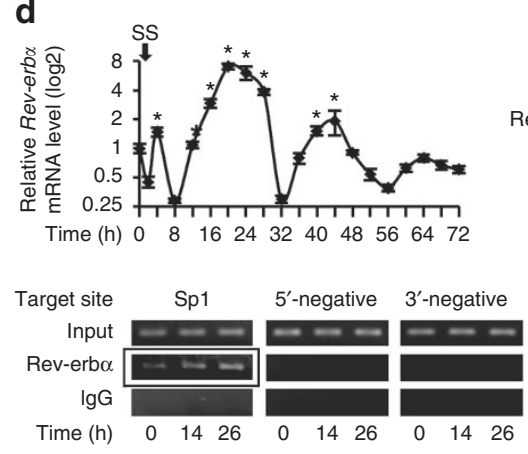
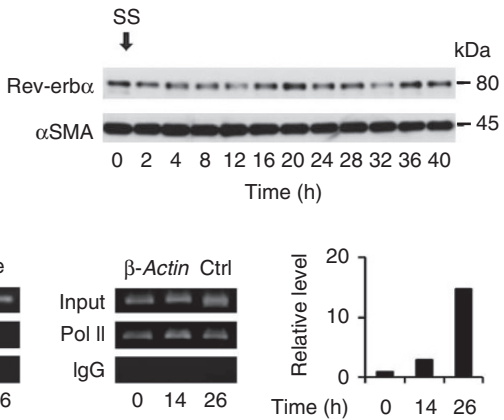

e
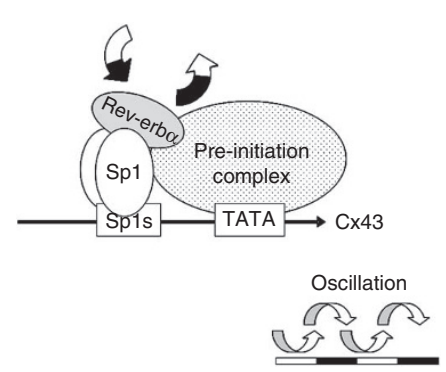

Figure 7 | Rhythmic assembly of Rev-erb $\alpha$ and Sp1 at Sp1 sites of the $\mathbf{C x 4 3}$ promoter. (a) Co-immunoprecipitation showing a complex formation between HA-tagged Rev-erb $\alpha$ and DDDDK-tagged Sp1 transfected in HEK293T cells, using antibodies for HA and DDDDK. One representative of three experiments with similar results is shown. (b) ChIP assay using antibodies for HA and DDDDK in HEK 293T cells transfected with HA-Rev-erb $\alpha$ and DDDDK-Sp1. Analyses by real-time RT-PCR are shown, targeted against endogenous Sp1 sites of the human Cx43 promoter and its negative control

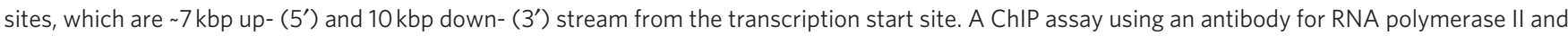
primers for human GAPDH promoter was used as a positive control. One representative of two experiments with similar results is shown. (c) Temporal Sp1 mRNA accumulation in the mouse bladder ( $n=3$ for each time point). There were no significant differences among time points by one-way ANOVA. (d) Oscillations of Rev-erb $\alpha$ mRNA (left) and protein expression (right) in serum-shocked rat BSMC (top row). ${ }^{*}<<0.01$ versus the nadir value (time 8 ) by one-way ANOVA with Dunnett's post hoc test $(n=3-6)$. SS, serum shock. The ChIP assay, using an antibody for endogenous Rev-erb $\alpha$, was analysed by RT-PCR targeted against endogenous Sp1 sites of the rat $C \times 43$ promoter and negative control sites, which are $~ 8 \mathrm{kbp}$ up- ( $5^{\prime}$-negative) and down( $3^{\prime}$-negative) stream (bottom row). $\beta$-Actin is a positive control. Results of real-time RT-PCR are added; it was targeted against Sp1 sites of the Cx43 promoter, which was immunoprecipitated using an antibody for Rev-erb $\alpha$ (corresponding to the framed bands, bottom). One representative of two experiments with similar results is shown. (e) A mechanistic scheme of Cx43 oscillation, controlled by the Rev-erb $\alpha$ and Sp1 complex binding to Sp1 sites of the $\mathrm{C} x 43$ promoter. Error bars represent s.d. in c, and s.e.m. in d. For relative levels, the maximal value was set as 1 in $\mathbf{c}$, and the values before serum shock (time 0 ) was set as 1 in $\mathbf{d}$.

\section{Methods}

Animals. Female $C x 43$ heterozygote KO mice $\left(C x 43^{+/-}\right)^{53}$ aged 16-21 weeks, their female WT littermates $\left(C x 43^{+/+}\right)$, and female Cry $1^{-1-}$ Cry $2^{-1-}$ mice (Crynull $)^{11}$ aged 10 weeks were used. C57BL/6 mice and Sprague Dawley (SD) female rats were purchased from Japan Lab Animals and Japan SLC. Animals were treated in accordance with NIH animal care guidelines, and the Kyoto University Animal Experiment Committee approved all animal experiments.

Micturition analysis in mice. Micturition assessment machines for aVSOP were manufactured by Real-designs(Kyoto, Japan). Rolled laminated filter paper, pre-treated to turn the edge of urine stains deep purple, was wound up at a speed of $10 \mathrm{~cm}$ per hour under a water-repellent wire lattice. Urine stains were counted and traced to convert micturition volume by the formula of a standard curve, calculated by the correlation of normal saline and the stained area ranging from $10-800 \mu \mathrm{l} . \mathrm{C} \times 43^{+/-}$and $\mathrm{C} \times 43^{+/+}$mice were kept in a cage with the dimensions of $110 \mathrm{~mm} \times 160 \mathrm{~mm} \times 75 \mathrm{~mm}$ (height $\times$ depth $\times$ width), measured under LD conditions for 4 days and killed for RNA extraction. The male WT mice were measured for 8 days under LD and followed by 5 days under DD conditions. The female WT and $C r y$-null mice were kept in a cage with dimensions of $75 \mathrm{~mm} \times 160 \mathrm{~mm} \times 75 \mathrm{~mm}$, and measured with a simultaneous actogram under DD conditions, for 5 days. Total urine volume per hour was estimated by dividing the volume by the time interval between the given and preceding voiding (filling time), when the filling time was more than $1 \mathrm{~h}$ (ref. 7).

Micturition analysis in rats. Micturition of SD rats was recorded by an electronic balance system ${ }^{22}$ under LD conditions for 2 days.

Real-time RT-PCR analysis. Female C57BL/6 mice aged 8 weeks and Cry-null mice aged 10 weeks were killed every $4 \mathrm{~h}$ at 6 time points during the day under a dim light ( $n=3$ for each time per strain) after acclimation for 2 weeks, under LD conditions followed by DD conditions. Female SD rats aged 7 weeks were acclimated for a week under LD conditions and killed at every $4 \mathrm{~h}$ ( $n=5$ for each time), followed by DD conditions, and then killed in the same manner $(n=2$ for each time). Complementary DNA was synthesized from $1 \mu \mathrm{g}$ of RNA extracted from the bladder and cultured cells, using a Superscript VILO cDNA Synthesis Kit (Invitrogen). Primers used are listed in Supplementary Table S1. Real-time RT-PCR was performed using SYBR Green PCR Master Mix with 7500 Fast Real-Time PCR system (Applied Biosystems) ${ }^{22}$. Each sample was normalized against an internal $18 \mathrm{~s}$ ribosomal RNA control (Takara). Maximal correlation coefficients (MaxCorr) were calculated using Mathematica ver. 5.1 according to a modified program ${ }^{35}$.

Immunoblotting. Whole-cell lysates from bladder tissues and cultured cells were lysed with radioimmunoprecipitation assay buffer containing proteinase inhibitors, which were resolved by SDS-PAGE and transferred to an Immobilon-P membrane. The membranes were incubated with antibodies against Cx43 (Zymed, 1:200; Sigma-Aldrich, 1:1000), Rev-erb $\alpha$ (Cell Signaling Technology, 1:500; Abcam, 1:600), Bmal1 (Santa Cruz, 1:200), Sp1 (Millipore, 1:2000), ఎSMA (Sigma-Aldrich, 1:5000), Sp3 (BioLegend, 1:500), HA (Abcam, 1:8000), DDDDK (MBL, 1:2000) and GAPDH (Cell Signaling, 1:2000). The immunoreactivities were visualized with enhanced chemiluminescence using HRP-conjugated anti-rabbit or mouse IgG antibody (Pierce) $)^{22}$.

Bioluminescence recording. Slice cultures of bladder were obtained from adult Period $2^{\text {Luciferase }}$ knock-in (Per2luc) mice ${ }^{38}$ (Jackson Laboratories), and Per2 ::luc mice with a Clock mutation $\left(\mathrm{Clk}^{\Delta 19} / \mathrm{Clk}^{\Delta 19}\right)$ generated by crossing Per $2:: l u c$ mice to Clockmutant $\left(\mathrm{Clk}^{\Delta 19} / \mathrm{Clk}^{\Delta 19}\right)$ mice (Jackson Laboratories). The slice cultures were kept at $36^{\circ} \mathrm{C}$ with culture medium containing $1 \mathrm{mM}$ luciferin ${ }^{54}$. Bioluminescence was measured with a highly sensitive cryogenic CCD camera (Spectra Video SV16KV/CT: PixelVision) equipped with a microscope (Carl Zeiss) $)^{55}$.

Immunostaining of the urinary bladder. Mice bladder tissues and slice cultures were fixed with $4 \%$ paraformaldehyde in $0.1 \mathrm{M}$. phosphate buffer (0.1MPB, $\mathrm{pH} 7.4$ ) for $24 \mathrm{~h}$ (ref. 54). After embedding bladder tissues in paraffin, we cut $5-\mu$ thick sections with a microtome. After being treated with $1 \%$ BSA, sections were incubated with Cx43 rabbit polyclonal antibody (Invitrogen, 1:50) or $\alpha$ SMA (Sigma-Aldrich, 
1:400). Immunoreactions were visualized by donkey anti-rabbit Alexa 594 (Molecular Probes), and observed by a fluorescent microscope (Carl Zeiss).

Serum shock analysis of BSMC. Primary BSMC, isolated from female SD rats aged 9 weeks ${ }^{22,56}$, were cultured after two passages until sub-confluent in DMEM with $10 \%$ FCS and $1 \%$ penicillin streptomycin followed by $72 \mathrm{~h}$ incubation in DMEM with $0.5 \%$ FCS. The cells were treated with $50 \%$ horse serum (GIBCO$\mathrm{BRL}$ ) in DMEM for $2 \mathrm{~h}$ (ref. 39), and washed twice with DMEM, and then maintained in DMEM with $0.5 \%$ FCS for a maximum of $72 \mathrm{~h}$.

Immunostaining of BSMC. BSMC were serum-shocked and fixed with $4 \%$ formaldehyde, permeabilized with $0.4 \%$ Triton X-100, and blocked with $10 \%$ goat serum (Invitrogen). The cells were incubated with $\mathrm{Cx} 43$ rabbit polyclonal antibody (Sigma-Aldrich, 1:500) followed by goat anti-rabbit Alexa 594 (Molecular Probes) and counter stained with 4,6-diamidino-2-phenylindole (DAPI) ${ }^{57}$

Lucifer yellow microinjections. Nuclei of serum-shocked BSMC were pre-stained with $16 \mu \mathrm{M}$ of Hoechst 33342 (Invitrogen) for $15 \mathrm{~min}$, to identify the site of injection. A single cell was impaled with a microelectrode, and Lucifer Yellow was injected by an electrometer (model 3100; A-M systems) for 1 min with a continuous current of $0.1 \mu \mathrm{A}$. Images were acquired using a CoolSNAP-HQ2 CCD camera (Photometrics $)^{57}$. Fluorescent cells were counted and the values were normalized by the total number of cells within the injected region of interest, with a mean cell density of $32.6 \pm 6.6$ cells).

Promoter-reporter assay. The promoter-reporter constructs used were, mouse pGL-2-Cx43 (pCx43 - 1686/ + 165-luc) ${ }^{42}$, mouse pGL-3-Per1 (pPer1-luc), pGL-3-Bmal1 (pBmal1-luc), and pGL-2 basic, pRL-TK (Promega) as controls. The expression vectors used were, $\mathrm{Sp} 1$ and $\mathrm{Sp} 3$ (fully lengthened according to a previous report ${ }^{58,59}$, Clock, Bmal1, Cry1, Dbp, E4bp4, Rev-erb $\alpha$ and Ror $\alpha$ (Open Biosystems). Site-directed mutagenesis, deletion and addition of aimed sequences were performed using a mutagenesis basal kit (Takara). Corrected mutants were all verified by sequencing. For luciferase assays, reporter plasmids $(100 \mathrm{ng})$ with various expression vectors (total $250 \mathrm{ng}$ ) were transfected to HEK293T cells in 24-well plates and those (total $100 \mathrm{ng}$ ) to BSMC in 96-well plates using Fugene6 (Roche) in DMEM with $10 \%$ FCS. Plasmid dosage was kept constant by the EGFP$\mathrm{N} 1$ vector. Lysates were collected $48 \mathrm{~h}$ post-transfection, and the luciferase activity was measured using a dual luciferase assay reagent (Promega).

RNA interference assay. Three sets of Stealth Select RNAi targeted against rat Rev-erb $\alpha$ (Rat Nr1d1 1330003) and Stealth RNAi Negative Control (low, medium and high GC, Invitrogen) were used. After two passages, BSMC were plated on 6-well plates and kept for $24 \mathrm{~h}$ in DMEM with $10 \%$ FCS. This was followed by transfection of siRNA (75 pmol per well) in serum-reduced $\alpha$ MEM for $5 \mathrm{~h}$ using Lipofectamin RNAiMAX (Invitrogen), followed by medium-change to DMEM with $10 \%$ FCS. After $48 \mathrm{~h}$ of the transfection, total RNA and cell lysates were extracted.

Co-immunoprecipitation assay. Rev-erb $\alpha$ with amino-terminal MYPYDVPDYAtag (HA-Rev-erb $\alpha$ ) and Spl with N-terminal MDYKDDDDK-tag (DDDDK-Sp1) were constructed using a mutagenesis basal kit (Takara). Nuclear extracts were prepared from HEK293T cells transfected with expression vectors for $48 \mathrm{~h}$, using a Nuclear Complex Co-IP kit (Active Motif). A total of $100 \mu \mathrm{g}$ of nuclear extracts were incubated with antibodies for $0.27 \mu \mathrm{g}$ of Rev-erb $\alpha$ (Cell Signalling), $4 \mu \mathrm{g}$ of HA (Abcam), DDDDK (MBL) and control rabbit IgG (Zymed), in $500 \mu \mathrm{l}$ of low IP buffer overnight at $4{ }^{\circ} \mathrm{C}$ with rotation followed by the addition of $30 \mu \mathrm{l}$ of Dynabeads Sheep anti-Rabbit IgG (Veritas) for $1 \mathrm{~h}$. After washing with low IP buffer with/without BSA three times each, the binding protein was eluted in $40 \mu \mathrm{l}$ of radioimmunoprecipitation assay buffer for immunoblotting. A total of $2 \mu \mathrm{g}$ of nuclear extracts were used as input.

Chromatin immunoprecipitation. Formaldehyde-crosslinked chromatins were obtained from HEK293T cells and serum-shocked BSMC, which were sheared using Chip-IT Express Enzymatic (Active Motif). Sheared chromatins were immunoprecipitated with antibodies to $0.23 \mu \mathrm{g}$ of Rev-erb $\alpha$ (Lifespan Biosciences, LS-C37817), $2 \mu \mathrm{g}$ of HA, DDDDK and control rabbit IgG overnight at $4{ }^{\circ} \mathrm{C}$ with rotation followed by the addition of $15 \mu \mathrm{l}$ of Dynabeads Sheep anti-Rabbit IgG for $1 \mathrm{~h}$. The Chip-IT control kits Rat and Human (Active Motif) were used as an experimental control. The enrichment of Sp1-binding sequences in eluted DNA was de-crosslinked by incubation at $65^{\circ} \mathrm{C}$, for $4 \mathrm{~h}$, followed by digestion of protein by proteinase K. Purified DNA, using a QIAquick PCR purification kit (Qiagen), was quantified by real-time RT-PCR and normalized by the quantity of input $\mathrm{DNA}^{60}$. Samples of BSMC after real-time RT-PCR were visualized by electrophoresis. Primers used are listed in Supplementary Table S1.

Microarray analysis of the mouse bladder. Whole bladder RNA of female C57BL/ 6 mice killed at CT $0,4,8,12,16$ and 20 as described above was used $(n=2$ for each time point). A total of $250 \mathrm{ng}$ of qualified RNA was employed for the synthesis of Cy3-labelled antisense RNA, which was qualified and hybridized to the Whole Mouse Genome Oligo Microarray (44K; Agilent Technologies) according to the manufacturer's protocol (entrusted to DNA Chip Research, Yokohama, Japan). Statistical analysis of microarray data was performed using R statistical software (version 2.8.1, 2008-12-22) with BioConductor (version 2.3). Signal processing was performed by Agilent Feature Extraction (version. 9.5.3). Normalization was performed based on the Agi4x44PreProcess package (version 1.2.0). The mgug4122a.db package (version 2.2.5) was used for annotation of each probe. Selection of circadian-oscillated genes was based on the modified procedure of Yamada et al. ${ }^{35}$ and McDonald et al. ${ }^{34}$. Specifically, we calculated correlation coefficients between each six-point time course and 60 different-phased cosine curves with six time points. For each probe, we selected one cosine curve with MaxCorr from the 60 different-phased cosine curves. The false-positive proportion was estimated on the basis of the null distribution of maximum correlations derived from randomly generated expression profiles.

Statistical analysis. For the micturition experiments, we used one-way ANOVA with Bonferroni's post hoc test to evaluate differences among time points. Two-way repeated measures ANOVA was used to compare differences between the two groups of mice tested, and time points were compared with Bonferroni's post hoc test. For the experiments in which three or more test groups were compared, we used one-way ANOVA, and for those including two factors, we used two-way ANOVA.

\section{References}

1. Robson, W. L. Clinical practice. Evaluation and management of enuresis. N. Engl. J. Med. 360, 1429-1436 (2009).

2. Neveus, T. Diagnosis and management of nocturnal enuresis. Curr. Opin. Pediatr. 21, 199-202 (2009).

3. Bosch, J. L. \& Weiss, J. P. The prevalence and causes of nocturia. J. Urol. 184, 440-446 (2010)

4. van Kerrebroeck, P. et al. The standardisation of terminology in nocturia: report from the Standardisation Sub-committee of the International Continence Society. Neurourol. Urodyn. 21, 179-183 (2002).

5. Van Hoeck, K. et al. Urine output rate and maximum volume voided in schoolage children with and without nocturnal enuresis. J. Pediatr. 151, 575-580 (2007).

6. Weiss, J. P., Blaivas, J. G., Stember, D. S. \& Chaikin, D. C. Evaluation of the etiology of nocturia in men: the nocturia and nocturnal bladder capacity indices. Neurourol. Urodyn. 18, 559-565 (1999).

7. Van Hoeck, K., Bael, A., Lax, H., Hirche, H. \& van Gool, J. D. Circadian variation of voided volume in normal school-age children. Eur. J. Pediatr. 166, 579-584 (2007).

8. Nakamura, S. et al. Circadian changes in urine volume and frequency in elderly men. J. Urol. 156, 1275-1279 (1996).

9. Witjes, W. P., Wijkstra, H., Debruyne, F. M. \& de la Rosette, J. J. Quantitative assessment of uroflow: is there a circadian rhythm? Urology 50, 221-228 (1997).

10. Reppert, S. M. \& Weaver, D. R. Coordination of circadian timing in mammals. Nature 418, 935-941 (2002).

11. Doi, M. et al. Salt-sensitive hypertension in circadian clock-deficient Cry-null mice involves dysregulated adrenal Hsd3b6. Nat. Med. 16, 67-74 (2010).

12. Takahashi, J. S., Hong, H. K., Ko, C. H. \& McDearmon, E. L. The genetics of mammalian circadian order and disorder: implications for physiology and disease. Nat. Rev. Genet. 9, 764-775 (2008).

13. Dibner, C., Schibler, U. \& Albrecht, U. The mammalian circadian timing system: organization and coordination of central and peripheral clocks. Annu. Rev. Physiol. 72, 517-549 (2010).

14. Panda, S. et al. Coordinated transcription of key pathways in the mouse by the circadian clock. Cell 109, 307-320 (2002)

15. Ueda, H. R. et al. System-level identification of transcriptional circuits underlying mammalian circadian clocks. Nat. Genet. 37, 187-192 (2005).

16. Okamura, H., Doi, M., Fustin, J. M., Yamaguchi, Y. \& Matsuo, M. Mammalian circadian clock system: molecular mechanisms for pharmaceutical and medical sciences. Adv. Drug Deliv. Rev. 62, 876-884 (2010).

17. Birder, L. et al. Neural control of the lower urinary tract: peripheral and spinal mechanisms. Neurourol. Urodyn. 29, 128-139 (2010).

18. Andersson, K. E. Antimuscarinic mechanisms and the overactive detrusor: an update. Eur. Urol. 59, 377-386 (2010).

19. Imamura, M. et al. Basic fibroblast growth factor causes urinary bladder overactivity through gap junction generation in the smooth muscle. Am. J. Physiol. Renal. Physiol 297, F46-F54 (2009).

20. Christ, G. J. et al. Increased connexin43-mediated intercellular communication in a rat model of bladder overactivity in vivo. Am. J. Physiol. Regul. Integr. Comp. Physiol. 284, R1241-R1248 (2003)

21. Suadicani, S. O., Urban-Maldonado, M., Tar, M. T., Melman, A. \& Spray, D. C. Effects of ageing and streptozotocin-induced diabetes on connexin 43 and P2 purinoceptor expression in the rat corpora cavernosa and urinary bladder. BJU Int. 103, 1686-1693 (2009). 
22. Negoro, H. et al. Regulation of connexin 43 by basic fibroblast growth factor in the bladder: transcriptional and behavioral implications. J. Urol. 185, 2398-2404 (2011)

23. Haferkamp, A. et al. Increased expression of connexin 43 in the overactive neurogenic detrusor. Eur. Urol. 46, 799-805 (2004)

24. Sugino, Y. et al. Voided stain on paper method for analysis of mouse urination. Neurourol. Urodyn. 27, 548-552 (2008).

25. Wood, R., Eichel, L., Messing, E. M. \& Schwarz, E. Automated noninvasive measurement of cyclophosphamide-induced changes in murine voiding frequency and volume. J. Urol. 165, 653-659 (2001).

26. Birder, L. A. et al. Altered urinary bladder function in mice lacking the vanilloid receptor TRPV1. Nat. Neurosci. 5, 856-860 (2002).

27. Reaume, A. G. et al. Cardiac malformation in neonatal mice lacking connexin43. Science 267, 1831-1834 (1995).

28. Bassuk, J. A., Grady, R. \& Mitchell, M. Review article: the molecular era of bladder research. Transgenic mice as experimental tools in the study of outlet obstruction. J. Urol. 164, 170-179 (2000).

29. Herrera, G. M. \& Meredith, A. L. Diurnal variation in urodynamics of rat. PLoS One 5, e12298 (2010).

30. Meredith, A. L. et al. BK calcium-activated potassium channels regulate circadian behavioral rhythms and pacemaker output. Nat. Neurosci. 9, 1041-1049 (2006)

31. van der Horst, G. T. et al. Mammalian Cry1 and Cry2 are essential for maintenance of circadian rhythms. Nature 398, 627-630 (1999).

32. Storch, K. F. et al. Extensive and divergent circadian gene expression in liver and heart. Nature 417, 78-83 (2002).

33. Hoogerwerf, W. A. et al. Transcriptional profiling of mRNA expression in the mouse distal colon. Gastroenterology 135, 2019-2029 (2008).

34. McDonald, M. J. \& Rosbash, M. Microarray analysis and organization of circadian gene expression in Drosophila. Cell 107, 567-578 (2001).

35. Yamada, R. \& Ueda, H. R. Microarrays: statistical methods for circadian rhythms. Methods Mol. Biol. 362, 245-264 (2007).

36. Fallon, R. F. \& Goodenough, D. A. Five-hour half-life of mouse liver gapjunction protein. J. Cell Biol. 90, 521-526 (1981).

37. Bennett, M. V. et al. Gap junctions: new tools, new answers, new questions. Neuron 6, 305-320 (1991).

38. Yoo, S. H. et al. PERIOD2::LUCIFERASE real-time reporting of circadian dynamics reveals persistent circadian oscillations in mouse peripheral tissues. Proc. Natl Acad. Sci. USA 101, 5339-5346 (2004).

39. Balsalobre, A., Damiola, F. \& Schibler, U. A serum shock induces circadian gene expression in mammalian tissue culture cells. Cell 93, 929-937 (1998).

40. Mitsui, S., Yamaguchi, S., Matsuo, T., Ishida, Y. \& Okamura, H. Antagonistic role of E4BP4 and PAR proteins in the circadian oscillatory mechanism. Genes Dev. 15, 995-1006 (2001).

41. Preitner, N. et al. The orphan nuclear receptor REV-ERBalpha controls circadian transcription within the positive limb of the mammalian circadian oscillator. Cell 110, 251-260 (2002)

42. Chen, Z. Q. et al. Identification of two regulatory elements within the promoter region of the mouse connexin 43 gene. J. Biol. Chem. 270 , 3863-3868 (1995)

43. Ueda, H. R. et al. A transcription factor response element for gene expression during circadian night. Nature 418, 534-539 (2002).

44. Oyamada, M., Oyamada, Y. \& Takamatsu, T. Regulation of connexin expression. Biochim. Biophys. Acta 1719, 6-23 (2005).

45. Teunissen, B. E. et al. Analysis of the rat connexin 43 proximal promoter in neonatal cardiomyocytes. Gene 322, 123-136 (2003).

46. Echetebu, C. O., Ali, M., Izban, M. G., MacKay, L. \& Garfield, R. E. Localization of regulatory protein binding sites in the proximal region of human myometrial connexin 43 gene. Mol. Hum. Reprod. 5, 757-766 (1999).

47. Boivin, D. B., Duffy, J. F., Kronauer, R. E. \& Czeisler, C. A. Sensitivity of the human circadian pacemaker to moderately bright light. J. Biol. Rhythms $\mathbf{9}$, 315-331 (1994).

48. Yeung, C. K., Diao, M. \& Sreedhar, B. Cortical arousal in children with severe enuresis. N. Engl. J. Med. 358, 2414-2415 (2008).

49. Yin, L. \& Lazar, M. A. The orphan nuclear receptor Rev-erbalpha recruits the $\mathrm{N}$-CoR/histone deacetylase 3 corepressor to regulate the circadian Bmall gene. Mol. Endocrinol. 19, 1452-1459 (2005).
50. Suzuki, Y. et al. Physical interaction between retinoic acid receptor and Sp1: mechanism for induction of urokinase by retinoic acid. Blood 93, 4264-4276 (1999).

51. Shimada, J. et al. Transactivation via RAR/RXR-Sp1 interaction: characterization of binding between Spl and GC box motif. Mol. Endocrinol. 15, 1677-1692 (2001).

52. Sun, G., Porter, W. \& Safe, S. Estrogen-induced retinoic acid receptor alpha gene expression: role of estrogen receptor-Spl complex. Mol. Endocrinol. 12, 882-890 (1998).

53. Suadicani, S. O., Brosnan, C. F. \& Scemes, E. P2X7 receptors mediate ATP release and amplification of astrocytic intercellular Ca2+ signaling. J. Neurosci. 26, 1378-1385 (2006)

54. Yamaguchi, S. et al. Synchronization of cellular clocks in the suprachiasmatic nucleus. Science 302, 1408-1412 (2003).

55. Doi, M. et al. Circadian regulation of intracellular G-protein signalling mediates intercellular synchrony and rhythmicity in the suprachiasmatic nucleus. Nat. Commun. 2, 327 (2011).

56. Wang, H. Z., Brink, P. R. \& Christ, G. J. Gap junction channel activity in short-term cultured human detrusor myocyte cell pairs: gating and unitary conductances. Am. J. Physiol. Cell Physiol. 291, C1366-C1376 (2006).

57. Thi, M. M., Urban-Maldonado, M., Spray, D. C. \& Suadicani, S. O. Characterization of hTERT-immortalized osteoblast cell lines generated from wild-type and connexin43-null mouse calvaria. Am. J. Physiol. Cell Physiol. 299, C994-C1006 (2010).

58. Hagen, G., Muller, S., Beato, M. \& Suske, G. Sp1-mediated transcriptional activation is repressed by Sp3. EMBO J. 13, 3843-3851 (1994).

59. Sapetschnig, A., Koch, F., Rischitor, G., Mennenga, T. \& Suske, G. Complexity of translationally controlled transcription factor $\mathrm{Sp} 3$ isoform expression. J. Biol. Chem. 279, 42095-42105 (2004).

60. Kanematsu, A., Ramachandran, A. \& Adam, R. M. GATA-6 mediates human bladder smooth muscle differentiation: involvement of a novel enhancer element in regulating alpha-smooth muscle actin gene expression. Am. J. Physiol. Cell Physiol. 293, C1093-C1102 (2007).

\section{Acknowledgements}

We thank D.C.Spray, A.Mello, M.M.Thi, M.Tanaka, R.Matsuoka, Y.Kimura, N.Kawakami, Y.Sugino, T.Kobayashi, Y.Kajita, S.J.Lye, J.Yao, G.Suske, J.Toguchida, M.Yamamoto, S.Karki, J.M.Fustin and A.Negoro. This work was supported by a Grant-in-Aid for Scientific Research $(20390425,21390439,23659756$ and 18002016) from the Japan Society for the Promotion of Science, a grant from the National Institutes of Health (DK 081435), a grant from the Translational Research Centre in Kyoto University, an Asah Kasei Pharma Urological Academy Grant, the Suzuki Urological Foundation and the Kyoto University Foundation.

\section{Author contributions}

H.N. and A.K. designed the experiments, analysed data and prepared the manuscript. H.N. performed most of the experiments. M.D. and H.O. contributed to the study design and manuscript preparation. S.O.S. provided $C x 43^{+/-}$mice and contributed to the microinjection of Lucifer yellow. M.M. contributed to the analysis of rhythm-associated experiments. M.I., T. Okinami, N.N., K.S., M.T. and S.U. helped with in vitro experiments T. Oura and S.M. analysed microarray data. E.K. contributed to morphological analysis. T.T. provided Cry-null mice. H.O., Y.T. and O.O. supervised the study.

\section{Additional information}

Accession codes: Microarray data have been deposited in the Gene Expression Omnibus under accession code GSE35795.

Supplementary Information accompanies this paper at http://www.nature.com/ naturecommunications

Competing financial interests: The authors declare no competing financial interests.

Reprints and permission information is available online at http://npg.nature.com/ reprintsandpermissions/

How to cite this article: Negoro, H. et al. Involvement of urinary bladder Connexin 43 and the circadian clock in coordination of diurnal micturition rhythm. Nat. Commun. 3:809 doi: 10.1038/ncomms1812 (2012). 